\title{
Foreword to the Special Issues in honor of Andrei Suslin on his 60th birthday
}

It is a great pleasure for the Editors of the Journal of K-Theory to congratulate their fellow Editor, colleague and friend Andrei Suslin on his 60th birthday and to wish him many more birthdays to come. For this occasion, the Journal of $K$-Theory is following a tradition begun by the earlier journal $K$-Theory to honor A. Grothendieck and D. Quillen on their 60th birthdays and is bringing out 2 special issues in honor of Andrei. This is the first issue. The second issue will appear later this year. We are also pleased to announce that the journal Documenta Mathematica will also honor Andrei this year with a volume of its journal.

Andrei's achievements and influence on the development of subjects served by our journal are enormous. We mention just a few of the highlights. His early work in the period 1974-1981 concerned projective, symplectic, and orthogonal modules and their automorphism groups. His solution in 1976 of the Serre conjecture on the freeness of projective modules over a polynomial ring over a field, established independently by Daniel Quillen in the same year, earned him the Komsomol Prize, the most prestigious honor for young scientists in the former Soviet Union. His paper also contained the analogous result for symplectic modules. One does not expect the same result for quadratic modules over a polynomial ring over a field (of characteristic not equal 2), but rather that such modules are extended. This result was proved in a joint paper with his student V.I. Kopeiko in 1977, under the condition that the Witt index of the module is at least 2. All of the above results are closely tied to the cancellation problem for modules over commutative rings and this was the topic of Andrei's talk at ICM 1978 in Helsinki. It would be the first of 3 ICM talks he was invited to give, the second of which would be a plenary talk. In the years immediately after the Helsinki meeting, his work included the normality of the elementary subgroup of a general linear group of rank at least 3 over a module finite ring (one which is finitely generated as a module over its center), the centrality of any Steinberg group extension of rank at least 4 over a module finite ring (joint with his student M.S. Tulenbaev), and his stability theorem for higher K-groups over rings of finite stable rank. The first and last paper of the current issue use ideas, techniques, and results from the early period above.

At the beginning of the 1980s, Andrei turned his attention to the K-theory of fields and division rings. By a classical result of Kummer, the norm residue homomorphism of degree 1 was known to be an isomorphism. In 1982, Andrei showed together with A. Merkurjev that the norm residue homomorphism of degree 
2 was also an isomorphism. This confirmed that the Bloch-Kato conjecture was true in degree 2 as well as degree 1. The Merkurjev-Suslin Theorem had many other important consequences, one of which was the solution of the long standing conjecture of A. A. Albert that every division algebra which is finite over its center $F$ and of exponent $n$ in the Brauer group $\operatorname{Br}(F)$, where $n$ is prime to the characteristic of $F$, is similar to a tensor product of cyclic division algebras over $F$ of degree $n$. At about the same time, he provided an ingeneous construction of a map $K_{i}(F) \rightarrow K_{i}^{M}(F)$ for all $\mathrm{i} \geq 1$ and computed for any infinite field $F$ both composite mappings of the map above with the map $K_{i}^{M}(F) \rightarrow K_{i}(F)$ induced by products. In the following year 1983, Andrei proved the Quillen-Lichtenbaum conjecture for algebraically closed fields, first in positive characteristic, extending Quillen's previous result for the algebraic closure of a finite field, and then for fields of characteristic zero. The conjecture states that for an algebraically closed field $F$, $K_{i}(F)$ is divisible if $i \geq 1$, the torsion being zero if $i$ is even and isomorphic to the $n$th Tate twist of the roots of unity in $F$ if $i$ is odd and $i=2 n-1$. The paper Torsion in $K_{2}$ of Fields began the first issue of the journal $K$-Theory. It establishes a complete cohomological description of the torsion in $K_{2}$ of fields of positive characteristic and finitely generated fields of characteristic zero, generalizing results of Tate, and constructs the reduced norm for $K_{2}$ of a finite, central, simple algebra. This was a very important ingredient in the K-theoretic solution of the Congruence and Metaplectic Problems for $S L_{n}(n \geq 2)$ of division rings which are finite over a global field. Andrei's 1986 ICM Plenary Address in Berkeley (delivered by E. Friedlander, since Andrei was not able to attend) was titled Algebraic K-theory of fields and included an overview of the results above.

In his talk at the Luminy conference on algebraic K-theory in 1987, Andrei proposed constructing a singular homology theory of abstract algebraic varieties (over arbitrary fields) as part of a program of computing the higher algebraic K-theory of varieties over the complex numbers $\mathbb{C}$. This theory should have two properties: Over $\mathbb{C}$, it should be identical with the usual homology of a variety and over arbitrary fields, it should yield the higher Chow groups of S. Bloch. In a joint paper with V. Voevodsky, the singular homology theory was constructed and shown to satisfy the first property above. The relation with higher Chow groups was established in his paper Higher Chow groups and étale cohomology. These results as well as the motivic cohomology theories being proposed in response to the Beilinson conjectures formed the core of his 1994 Zurich ICM talk and included a solution of the Quillen-Lichtenbaum conjecture for curves and surfaces. The motivic cohomology theory described in the talk was subsequently applied in a joint paper published in 2000 with Voevodsky titled Bloch-Kato conjecture and motivic cohomology with finite coefficients to show that the Bloch-Kato conjecture implied 
the Beilinson-Lichtenbaum conjecture.

During the late 1990s, Andrei was a coauthor of a remarkable series of papers with E. Friedlander, studying the cohomology ring of group schemes. In one paper, the cohomology ring of a finite group scheme was shown to be finitely generated. In papers with C. Bendel, they provided a concrete realization of support varieties for infinitesimal group schemes. And in a paper with V. Franjou and A. Scorichenko, they developed a powerful functor category method for computing Ext groups over the infinite general linear group of a finite field. This is useful for computing motivic cohomology groups with finite coefficients.

For the body of work mentioned in the previous two paragraphs, Andrei Suslin received the 2000 Cole Prize in Algebra.

This overview of highlights of Andrei's contributions to K-theory, (co)homology theory, and algebraic geometry is not exhaustive. In an important paper in 1992 , Andrei and M. Wodzicki characterized those $\mathbb{Q}$-algebras that satisfy excision in algebraic K-theory, and used this to prove a conjecture of M. Karoubi on algebraic $\mathrm{K}$-theory of stable $C^{*}$-algebras. In the last decade, Andrei has produced other remarkable results in the above areas of mathematics. We mention three items. One is his work on the spectral sequence from motivic cohomology to algebraic $K$-theory. Another is his joint paper with S. Joukhovitski entitled Norm Varieties which presented an account of M. Rost's construction of certain splitting varieties; this construction was needed in Voevodsky's proof of the Bloch-Kato conjecture, and hence of the Lichtenbaum-Quillen conjecture. The third is his 2006 paper $S K_{1}$ of division algebras and Galois cohomology revisited. This topic and paper form the focus and background of the third article of our tribute to Andrei.

May 2010

Anthony BaK, Jonathan Rosenberg ANd ChaRles Weibel

Managing Committee of the Journal of K-Theory 